\title{
Coordinating the interaction between past and present: Visual working memory for feature bindings overwritten by subsequent action to matching features
}

\author{
Chris M. Fiacconi ${ }^{1} \cdot$ Jessica N. Cali ${ }^{2} \cdot$ Juan Lupiáñez $^{3} \cdot$ Bruce Milliken $^{2}$ \\ Published online: 24 December 2019 \\ (C) The Psychonomic Society, Inc. 2019
}

\begin{abstract}
The object-file framework forwarded by Kaheman, Treisman, and Gibbs (1992) has been enormously influential in our understanding of how the visual system links together prior visual content with a current input. Although this framework was initially developed to account for the perceptual benefits associated with feature conjunction repetitions, the present series of experiments examines how the core processes of this framework may also help explain behavior in tasks that require explicit remembering of visual information over the short term. Building off our previous work (Fiacconi \& Milliken, 2012, 2013), here we introduce a procedure that affords the opportunity to examine the contributions of object-file review processes to both speeded performance and visual short-term memory (VWM) within the same task. Across two experiments we demonstrate a novel coupling between memory accuracy and speeded performance, such that the conditions that promote faster performance also tend to produce better memory, and vice versa. These findings are discussed in relation to the object-file framework and suggest that the object-file review processes known to guide behavior in speeded performance tasks may also have important mnemonic consequences. Together, these findings unite two lines of research to which Anne Treisman made indelible contributions.
\end{abstract}

Keywords Attention: interactions with memory · Visual working memory

Humans can access representations of visual experience for a period of time after the corresponding visual events terminate. Our ability to accomplish this feat is thought to rely on retrieval from a visual working memory (VWM) system that stores items for a relatively brief duration. VWM is often studied by presenting a memory array followed by a probe - the role of the probe is, ostensibly, to measure what has been retained in VWM (Luck \& Vogel, 1997; Wheeler \& Treisman, 2002). However, a challenge with this method is that probes following a memory array can also be integrated with representations of spatially corresponding memory array items (Averbach

Chris M. Fiacconi

cfiaccon@uoguelph.ca

1 Department of Psychology, University of Guelph, Guelph, Ontario, Canada

2 Department of Psychology, Neuroscience, \& Behavior, McMaster University, Hamilton, Ontario, Canada

3 Facultad de Psicología, Universidad de Granada, Granada, Spain
\& Coriell, 1961). This integration between memory array and subsequent probe can complicate inferences about VWM, as the tool used to measure VWM properties (the probe) itself interferes with VWM performance.

Whereas spatiotemporal integration of probes with memory representations has been an obstacle in studies of VWM, it was the direct target of study in the object file review work of Kahneman, Treisman, and Gibbs (1992). Kahneman et al. proposed that integration of current perceptual representations with memory representations of recent prior events is a ubiquitous process that ensures we see objects as continuous across time. They reported a series of experiments in which a preview array was followed by a probe that participants were required to identify. The results strongly implicated an integration process involving the probe and spatiotemporally corresponding items in the preview array.

Although studies of both VWM and object file review imply a process in which probes are integrated with memory representations of immediately preceding events, to our knowledge no study has yet combined VWM and object review methods to study this process. In the present study, we describe results from two experiments that combined a VWM 
method with a simple performance task that allowed us to measure how short-term remembering from VWM depends on the object file updating processes identified by Kahneman et al. (1992). A brief summary of the literature on short-term retention of features and objects is provided below, prior to describing the specific aims of the present study.

\section{Retention of feature bindings in VWM}

An issue that has received considerable study is whether information is stored in VWM as a collection of individual features, or as bound combinations of features known as objects (Delvenne \& Bruyer, 2004; Luck \& Vogel, 1997; Saiki, 2003; Vogel, Woodman, \& Luck, 2001; Wheeler \& Treisman, 2002; Xu, 2002). In a seminal study, Luck and Vogel used a change-detection procedure to examine this issue. Participants viewed an initial stimulus array (the memory array) containing a set of four squares. Each square consisted of a small inner square of one color superimposed on a large outer square of another color. Across conditions, participants were instructed to remember the colors of both the large outer and small inner squares, just the large outer squares, or just the small inner squares. Following the memory array a test display consisting of four squares appeared. On same trials, the four squares in the test display were identical to those in the memory array. On different trials, the color of one of the large or small squares changed to a new color relative to the memory array. Participants were asked to indicate whether the memory array and test display were the same or different. A key finding was that performance in the condition in which both large and small squares were to be remembered was equal to performance in the conditions in which only the large or only the small squares were to be remembered. This finding suggests that the conjunction of features that make up an object are stored as efficiently as the individual features themselves. Luck and Vogel interpreted these results as evidence for object-based storage in VWM.

In a subsequent study, Wheeler and Treisman (2002) reported results that contradicted this view (see also Delvenne \& Bruyer, 2004). They found that when test displays contained as many elements as the memory array (the whole-display condition), memory for feature bindings was impaired relative to memory for individual features. In contrast, when test displays contained only a single element (the single-probe condition), there was no evidence of a binding-specific memory deficit. To explain this pattern of results, Wheeler and Treisman (2002) proposed that maintenance of feature bindings in VWM does not "come for free," but rather requires focused attention. They proposed that in the whole display condition, attention was required to correctly bind together the features of multiple items in the test display (Treisman \& Gelade, 1980), thus diverting attention away from maintaining the memory array feature bindings. In contrast, when only a single item appeared in the test display, correct perception of this lone item required fewer attentional resources than in the whole display condition, and thus left more resources available to maintain the memory array feature bindings.

Yet this interpretation, too, was challenged (Gajewski \& Brockmole, 2006; Johnson, Hollingworth, \& Luck, 2008). Johnson et al. had one group of participants perform a demanding visual search during the retention interval of a change-detection task, whereas another group was not required to perform this search task. If attention is required to maintain feature bindings in VWM, then any binding-specific memory impairment should be larger for the visual search group. Contrary to this reasoning, the binding-specific impairment was no different for the two groups.

A potential resolution to the mixed evidence presented above hinges on a reinterpretation of the binding-specific memory deficits observed in the whole display condition of Wheeler and Treisman (2002). This reinterpretation assumes that feature bindings for memory array items are susceptible to overwriting upon onset of the test display (Allen, Baddeley, \& Hitch, 2006; Alvarez \& Thompson, 2009; Gorgoraptis, Catalao, Bays, \& Husain, 2011; Makovski, Sussman, \& Jiang, 2008; Makovski, Watson, Koutstaal, \& Jiang, 2010; Ueno, Allen, Baddeley, Hitch, \& Saito, 2011a; Ueno, Mate, Allen, Hitch, \& Baddeley, 2011b). Specifically, Wheeler and Treisman used test displays in the whole display condition that contained either the same objects as in the memory array (same trials), or the same features recombined into different conjunctions than in the memory array (different trials). Moreover, test display items appeared in the same spatial locations as the memory array items. Given the perfect feature overlap between the memory array and test display, the test display may have been treated as a "perceptual update" of the memory array, with this update effectively overwriting the initial feature bindings and rendering them inaccessible.

This overwriting account invokes many of the principles of the object file review framework developed by Kahneman et al. (1992). Kahneman et al. measured naming latencies for target letters that followed the presentation of two or more preview letters. Performance was particularly fast for target letters that matched the preview letter in both identity and location, but relatively slower for target letters that matched the location, but not the identity of the preview letter. This effect is known as the object-specific preview benefit. To account for this effect, Kahneman et al. suggested that a spatiotemporal match between a target letter and a preview letter triggers the updating of an object file. Object files are temporary, episodic memory representations that include feature bindings of previous events, and that can be updated with new perceptual features, thus continuously tracking the state of an object across time. It has been suggested that a similar updating process could occur with a VWM method such as 
change detection (Treisman \& Zhang, 2006; Wheeler \& Treisman, 2002). In particular, a spatiotemporal correspondence between the memory array and test display could induce an updating process that overwrites memory array feature bindings with new test display feature bindings. This overwriting process may then render the memory array bindings inaccessible (see also Pertzov, Dong, Peich, \& Husain, 2012).

An important implication of this overwriting account is that test displays in change detection tasks do not offer a pristine measure of the content of VWM. Rather, onset of a test display cues an interaction with VWM representations, and performance captures the outcome of this interaction. This idea fits with results of recent studies that have shown feature bindings to be "fragile" and readily disrupted by subsequent perceptual input (Allen et al., 2006; Alvarez \& Thompson, 2009; Gorgoraptis et al., 2011; Makovski et al., 2008; Makovski et al., 2010; Pertzov et al., 2012; Ueno, Allen, et al., 2011; Ueno, Mate, et al., 2011; Wheeler \& Treisman, 2002).

\section{Joint measurement of VWM and object file review}

Although the object file framework featured prominently in prior research on VWM by Treisman and others (Hollingworth \& Rasmussen, 2010; Treisman \& Zhang, 2006; Wheeler \& Treisman, 2002), the relation between feature binding and updating processes proposed to operate in VWM and those thought to mediate object file review effects in speeded performance (Kahneman et al., 1992) remains unclear. To study this relation directly, a method is needed that includes both speeded performance and memory components. We recently introduced such a method (Cali, Fiacconi, \& Milliken, 2015; Fiacconi \& Milliken, 2012, 2013). Briefly, participants were presented with a memory array of two items displayed in two of four marked locations. Memory for the location of one of these items was tested at the end of each trial. This was the VWM component of the task. Critically, following the memory array and prior to the VWM task at the end of the trial, an intervening display appeared. This intervening display also consisted of two items in two of the four marked locations, and it required a response to one of those items. We were particularly interested in VWM performance for memory array items that appeared in the same spatial location as a following intervening display item, as the spatial correspondence between these items offered the opportunity to study the integration processes of interest. A key finding from our prior studies was that VWM performance was particularly poor when participants responded to an intervening display item that mismatched the identity of the memory array item that appeared at the same spatial location.

\section{The present study}

In prior studies with this method, our primary focus was on how VWM performance is impacted by actions made in response to the intervening display. We were motivated to study this issue by Hommel's (1998) insight that both perceptual features and actions can be integrated into temporary episodic representations, which he called "event files" rather than "object files." Indeed, our research revealed that spatially directed responses to items in the intervening display moderated VWM performance; that is, actions can contribute to the "overwriting" of memory array items that limits VWM performance.

Our focus in the present study is more directly on the relation between object file review processes and VWM performance. As such, a key goal of our method is to measure the influence of object file updating processes on both speeded identification of intervening display items and VWM performance for memory array items. A close correspondence between these two measures would suggest a common set of object updating processes contribute to VWM and object file review, and unite two distinct lines of research to which Anne Treisman made indelible contributions (Kahneman et al., 1992; Treisman \& Zhang, 2006; Wheeler \& Treisman, 2002).

\section{Experiment 1}

To examine this potential correspondence, we used a method that was similar to that in our prior studies (Cali et al., 2015; Fiacconi \& Milliken, 2012, 2013), but in which the target identity in the intervening display was cued randomly from trial to trial. This procedure is depicted in Fig. 1. Cueing the intervening display target identity randomly on each trial ensured that participants were unaware of the target letter at the time the memory array was presented, and that participants were forced to selectively attend to the intervening display in order to determine the target identity for a given trial. A key feature of this procedure is that both memory for location-identity feature conjunctions and the reaction times (RTs) to the target identity in the intervening display could be measured together on a trialby-trial basis, affording the opportunity to explore the correspondence between memory accuracy and speeded performance. By requiring participants to execute speeded responses to target items in the intervening display, we were able to examine RTs to targets (a) that appeared in a location that had been unoccupied in the memory array (control trials); (b) that matched the identity of the item that had appeared at the same location in the memory array (match trials); and (c) that mismatched the identity of the item that had appeared at the same location in the memory array (mismatch trials). Note that this design parallels that used by Kahneman et al. in their pioneering work on object files. See Table 1 for a visual depiction of these key conditions. 


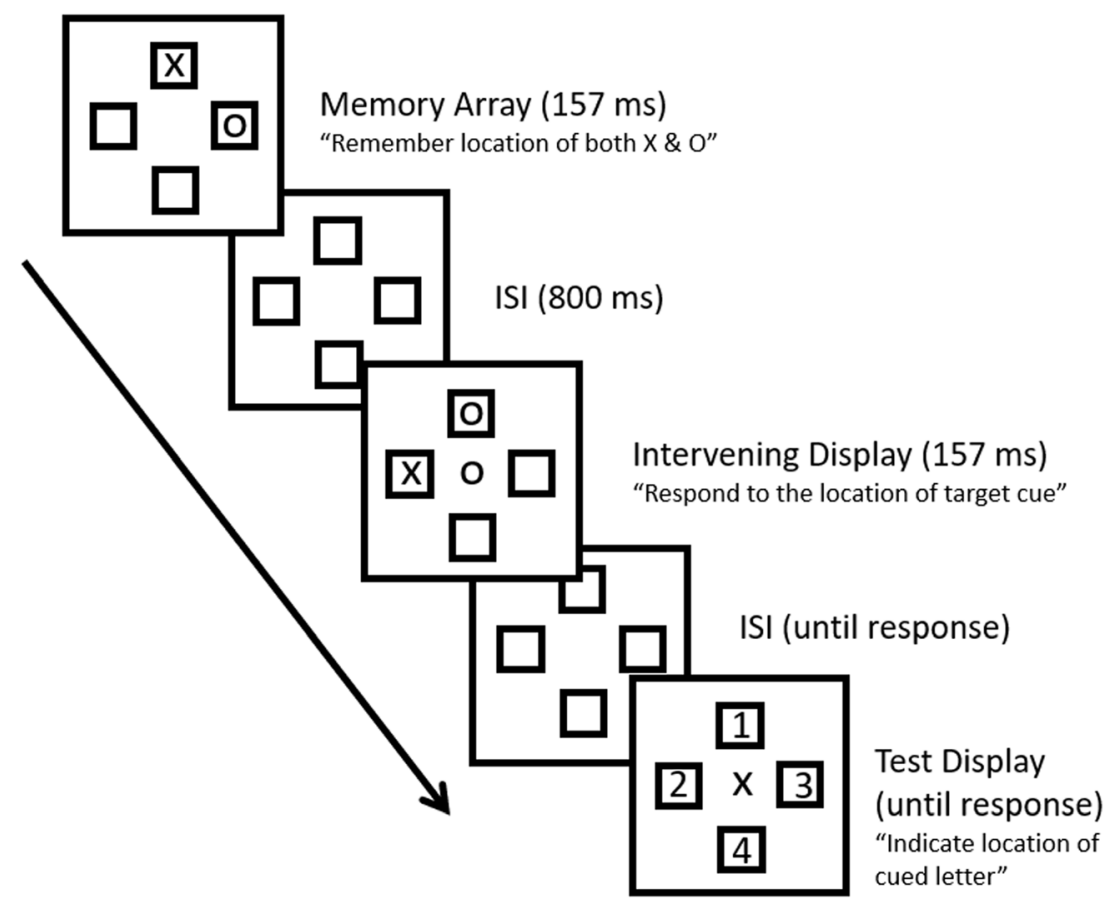

Fig. 1 Experimental procedure used in Experiment 1. Experiment 2 in the present article uses a variation of this basic procedure (see the text)

Table 1 Visual illustration of the experimental and control conditions included in the present experiments
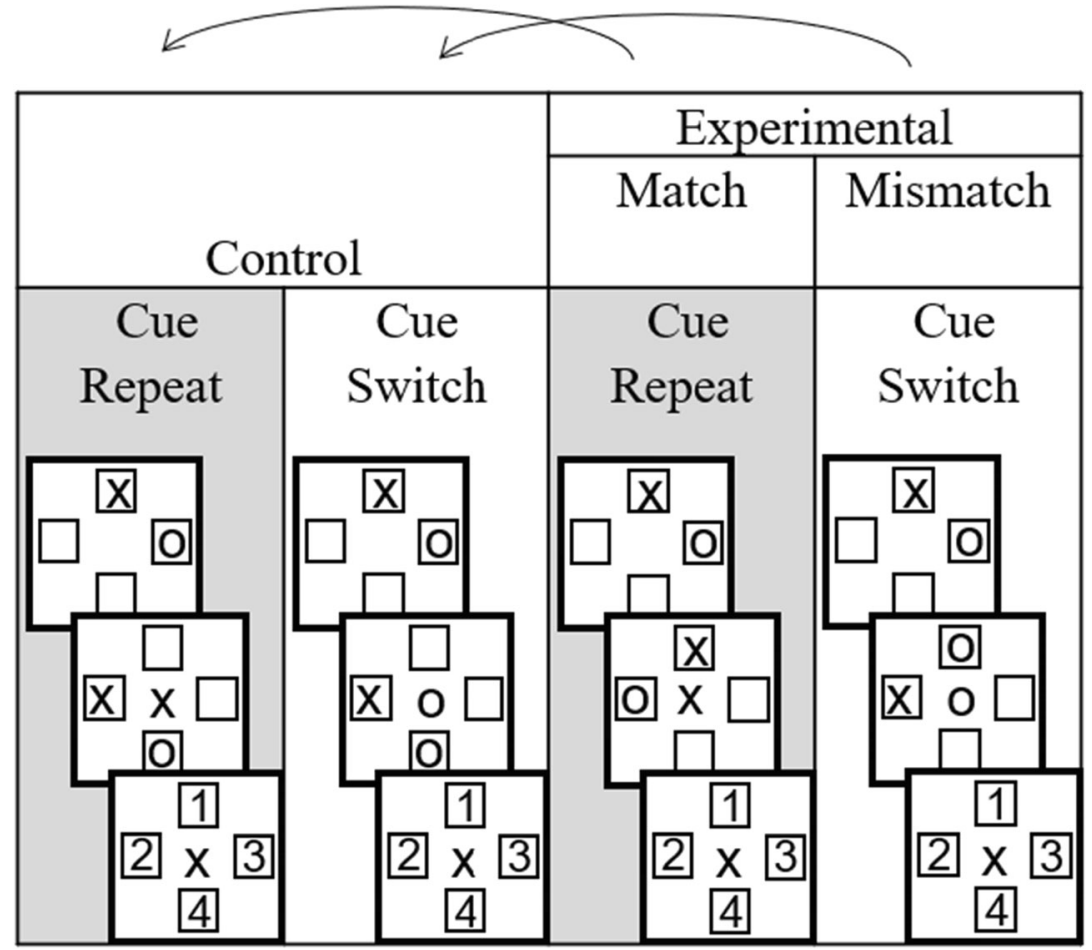

The control conditions represent trials in which no occupied locations were repeated between the memory array and the intervening display, whereas the experimental conditions are those in which the intervening display target appears in a location that was occupied in the memory array. The cue repeat/cue switch variable describes the relation between the identities of the intervening display target and memory probe, which can either repeat (same identity) or switch (different identity) on a given trial. Mnemonic costs were calculated by subtracting memory performance in the appropriate experimental condition from that in each control condition. RT costs were calculated by subtracting the RTs in the appropriate control from those in the appropriate experimental condition. 


\section{Method}

Participants The participants were 20 McMaster University undergraduate students (15 females, five males) who participated in exchange for course credit or financial compensation. The mean age of the participants was 18.8 years. All participants reported normal or corrected-to-normal vision.

Stimuli and apparatus The experiment was conducted on a Pentium IBM compatible computer equipped with a NEC MultiSync color monitor. Participants were seated approximately $40 \mathrm{~cm}$ from the monitor. Responses were made with a standard keyboard interfaced with the computer. Response times were measured using the procedure outlined in Bovens and Brysbaert (1990).

On each trial, four boxes were presented on the screen in light grey. The boxes were positioned such that the horizontal angle between the centers of the left and right boxes was $5.0^{\circ}$ and the vertical visual angle between the centers of the top and bottom boxes was $4.3^{\circ}$. The boxes themselves subtended a visual angle of $1.6^{\circ}$ horizontally and $1.7^{\circ}$ vertically. Two letters (the letter " $\mathrm{O}$ " and the letter " $\mathrm{X}$ ") appeared in two of the four possible marked locations, both in the memory array and in the intervening display. Both letters were presented in light gray, and subtended a visual angle of $0.9^{\circ}$ horizontally and $1.0^{\circ}$ vertically. In addition, for the intervening display, a green target cue letter (either an $\mathrm{X}$ or an $\mathrm{O}$ ) was presented in the center of the four boxes. In the test display, the numbers 1-4 appeared in the four boxes, and a memory cue (either an X or an $\mathrm{O}$ ) was positioned in the center of the display appearing in light grey.

Design Although a total of ten conditions were included in the experiments reported here, ${ }^{1}$ only four key conditions directly addressed our primary questions of interest. In the interest of brevity and clarity, we will focus our analyses on these four critical conditions. The four key conditions in the design are displayed in the heading structure of Table 1 . These four conditions can be divided into two broad categories: (1) control trials, in which there was no spatial overlap between the

\footnotetext{
${ }^{1}$ The full design consisted of ten conditions. In addition to the four conditions described in text, we also varied the intervening display target such that on half the trials the target appeared in a location previously unoccupied in the preceding memory array. We also varied which item from the memory was tested such that on half the trials we tested location memory for the item that was not overlapped by an item in the intervening display. Therefore, in addition to the two control conditions, the remaining eight experimental conditions resulted from the $2 \times 2 \times 2$ factorial combination of the match $/$ mismatch factor described in text with these other two additional manipulations. Of the resulting eight experimental conditions, we focus our analyses in the text on the match and mismatch trials in which the intervening display target appeared in a location that was repeated from the memory array and in which memory for the item that was subsequently overlapped in the intervening display was tested. The latter two experimental conditions were the ones most relevant for addressing our primary question of interest.
}

memory array and the intervening display items, and (2) experimental trials, in which one of the intervening display items overlapped spatially with a memory array item. The experimental trials can then be further subdivided into match trials and mismatch trials. On match trials, the intervening display target matched in both location and identity a memory array letter from the preceding memory array. On mismatch trials, the intervening display target matched a memory array letter from the preceding memory array in location, but not identity. Because our primary interest centered on the mnemonic and performance consequences associated with spatial correspondence between items in the memory array and intervening display, we subtracted performance for experimental trials from control trials. This subtraction results in a "cost" score that can be computed both in terms of memory accuracy (i.e., "mnemonic cost") and speeded performance (i.e., "RT cost"). These cost scores capture the extent to which VWM accuracy and RT are influenced by spatial correspondence between items in the memory array and the subsequent intervening display.

The reader will also notice that there are two control conditions. Note that a consequence of cueing the identity of the intervening display target and the identity of the memory probe independently is that for half of the trials, the identity of the intervening display target and the identity of the memory probe will match (cue repeat trials), and for the other half of trials they will mismatch (cue switch trials). This distinction is depicted in Table 1. Note that the two control conditions differ on this dimension; one is a cue switch trial and the other is a cue repeat trial. The two experimental conditions can similarly be split into cue switch and cue repeat trials. When computing the mnemonic costs defined above, it was important that the difference scores between control and experimental trials were computed using conditions in which the identity relation between the intervening display target and the subsequent memory probe was held constant.

All experimental conditions were presented mixed together, in six blocks of 48 trials each. Participants were given a forced 1-min rest period after each block of 48 trials, as well as the opportunity to rest whenever necessary between the trials within each block. Participants completed 24 practice trials prior to beginning the experimental session.

Procedure The trial sequence for Experiment 1 is depicted in Fig. 1. Each trial began with presentation of the four empty boxes for $1,000 \mathrm{~ms}$, followed by the memory array for $157 \mathrm{~ms}$. Participants were instructed to remember the locations of both letters $(\mathrm{X}$ and $\mathrm{O})$ in the memory array. The memory array was followed by an interstimulus interval (ISI) of $800 \mathrm{~ms}$, during which the four boxes were empty. The intervening display was then presented for $157 \mathrm{~ms}$. Participants were instructed to respond to the location of the letter that matched the green cue letter $(\mathrm{X}$ or $\mathrm{O})$, which appeared in the center of the display. 
This cue was equally often an X or an O. Participants recorded their responses to the intervening display using a keyboard, with "W" mapped to the top location, "S" mapped to the bottom location, "J" mapped to the left location, and "K" mapped to the right location. Participants heard a brief click when a correct response had been made, and they heard a brief, high-pitched tone when an incorrect response had been made. Immediately after the response to the intervening display, the test display appeared. In the test display, each of the four boxes was numbered 1-4, and a memory cue (either an X or an $\mathrm{O}$ ) was presented in the center of the screen. Participants were required to indicate by keypress, using the same response mappings as for the intervening display, the location at which the memory probe letter had appeared in the memory array. Memory for the location of each of the two letters was tested equally often (see note 1), although of greatest interest here were those trials in which the participant was asked to remember the location of an identity that had subsequently overlapped in the intervening display. As such, only these trials were included in our analysis. Responses to the test display were not speeded, but participants were instructed to try to respond within $3 \mathrm{~s}$. After the response to the test display, the screen was cleared, and participants initiated the next trial at their own pace with a press of the space bar.

\section{Results and discussion}

Memory analyses Only trials on which a correct response was made to the target in the intervening display were included in the memory analyses. The raw mean proportions correct for all four conditions can be found in Table 2 . The primary memory analyses were conducted on the mnemonic costs for the two experimental conditions. The means of the mnemonic costs for each condition, collapsed across participants, are displayed in Fig. 2.

To probe whether match and mismatch trials were associated with a significant mnemonic cost, we compared the cost scores for these trials against zero. The analyses revealed significant mnemonic costs for both the mismatch condition, $t(19)=7.71, p<.001, d_{\text {cohen }}=1.72$, and the match condition,

Table 2 Raw mean proportions of correct responses to the test display in Experiments 1 and 2

\begin{tabular}{llllll}
\hline Experiment & \multicolumn{2}{l}{ Control Trials } & & \multicolumn{2}{l}{ Experimental Trials } \\
\cline { 2 - 3 } \cline { 5 - 6 } \cline { 5 - 5 } & Cue Repeat & Cue Switch & & $\begin{array}{l}\text { Match } \\
\text { (Cue Repeat) }\end{array}$ & $\begin{array}{l}\text { Mismatch } \\
\text { (Cue Switch) }\end{array}$ \\
\hline 1 & .74 & .66 & .53 & .37 \\
2 & .94 & .43 & & .94 & .09 \\
\hline
\end{tabular}

The calculation of mnemonic cost for each experimental trial can be derived by subtracting the appropriate control condition, as displayed in Table 1

\section{Experiment 1 Accuracy}

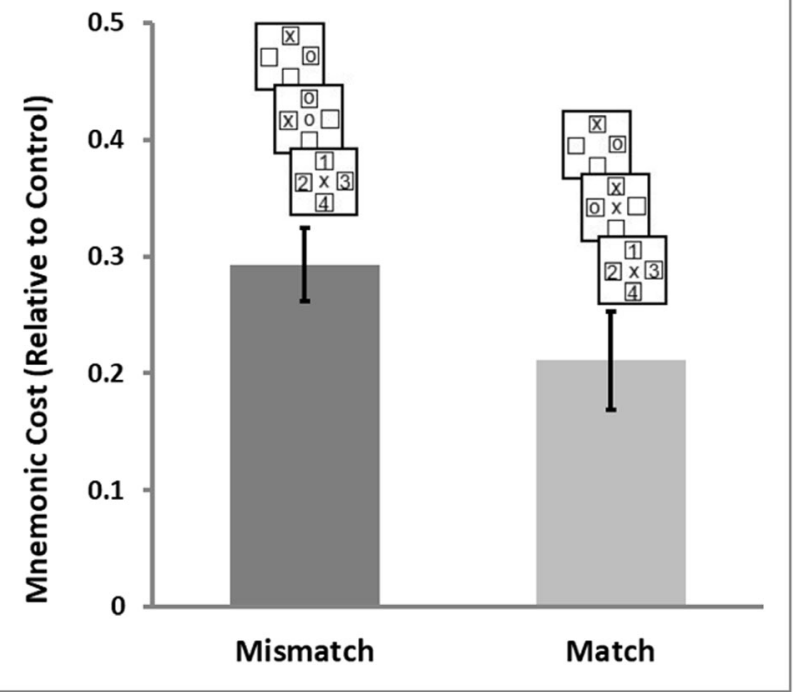

Fig. 2 Mean memory accuracy difference scores for the two conditions of interest in Experiment 1. Error bars represent the standard errors of the mean difference scores. Trial diagrams are shown above each bar for the relevant condition, where each large square represents a possible configuration for the memory array, intervening display, and test display in that trial type. The intervening item cue is shown in the center of the intervening display. Match and mismatch refer to whether the spatially overlapped item matches or mismatches between the memory array and the intervening display

$t(19)=4.99, p<.001, d_{\text {cohen }}=1.12$, but no significant difference between the costs for the match and mismatch trials, $t(19)=1.40, p=.18, d_{\text {cohen }}=0.46$. The observed mnemonic cost for match trials is particularly intriguing, as it points to a source for such costs that is not captured well by the object-file framework (Kahneman et al., 1992).

RT analyses A second measure of interest in this study was the time required to localize the intervening display target. Correct RTs were submitted to an outlier procedure (Van Selst \& Jolicœur, 1994) that excluded 3.2\% of the RTs from further analysis. Mean RTs were computed from the remaining observations, and are displayed for each of the three conditions in Table 3. The corresponding error rates are presented in Table 4. These error rates were analyzed but produced no significant effects (all $p \mathrm{~s}>.56$ ).

Similar to the memory accuracy analyses reported above, our primary interest was to measure the performance consequences of spatial correspondence between items in the memory array and the intervening display. For the RT analyses, we calculated difference scores by subtracting the mean RT for control trials from the mean RT for each of the experimental trials. We call these difference scores "RT costs." Positive RT costs indicate slower responses for experimental than for control conditions. The mean RT costs are displayed in Fig. 3. 
Table 3 Raw mean reaction times (RTs) for each condition in Experiments 1 and 2

\begin{tabular}{llll}
\hline Experiment & \multirow{2}{*}{ Control Trials } & \multicolumn{2}{l}{ Experimental Trials } \\
\cline { 3 - 4 } & & Match & Mismatch \\
\hline 1 & 913 & 943 & 972 \\
2 & 910 & 870 & 984 \\
\hline
\end{tabular}

Note that the cue repeat/switch variable is not relevant here, given that the RTs to targets are measured for the intervening display only. Therefore, the relationship between the identity of the intervening display target and the subsequent memory probe is irrelevant for this measure.

For the mismatch condition, the RT cost was significantly greater than zero, $t(19)=3.66, p=.002, d_{\text {cohen }}=0.82$, whereas for the match condition, the RT cost only approached significance, $t(19)=1.82, p=.08, d_{\text {cohen }}=0.41$. However, we found no reliable difference in the RT costs for the mismatch and match conditions, $t(19)=1.36, p=.19$.

Summary The key results here were the apparent mnemonic and RT costs observed for the match condition. Although the RT cost only approached significance, it demonstrates that, relative to the appropriate control condition, responses were slowed to the intervening display target when it matched both the location and identity of an item from the memory array. This result represents a marked departure from the findings of Kahneman et al. (1992), in which target items were identified faster when they matched in location and identity a preceding letter from the preview display (i.e., an object-specific preview benefit). That we observed an RT cost for match trials implies that the object-file updating processes identified by Kahneman et al. may not be obligatory, and are instead subject to contextual control. Moreover, the observed RT cost for match trials was accompanied by a corresponding mnemonic cost, such that memory performance could also be relatively poor for items that were followed by a subsequent target that matched in both location and identity. We will return to a more in-depth discussion of these issues in the General Discussion.

\section{Experiment 2}

One particularly compelling aspect of the results from Experiment 1 was the significant mnemonic cost in the match condition. ${ }^{2}$ Although we had observed a small effect of this type in one prior experiment (Fiacconi \& Milliken, 2013, Exp. 2 ), visual memory costs were otherwise specific to mismatch

\footnotetext{
${ }^{2}$ Due to the novelty of the results of Experiment 1, we ran a nearly identical experiment with a new set of participants. The only difference between this experiment and Experiment 1 was that the ISI between the memory array and intervening display was $500 \mathrm{~ms}$ (similar to that in Exp. 2). The results of this experiment completely replicated those reported in Experiment 1, adding confidence to our findings reported in that experiment.
}

\section{Experiment 1 Response Time}

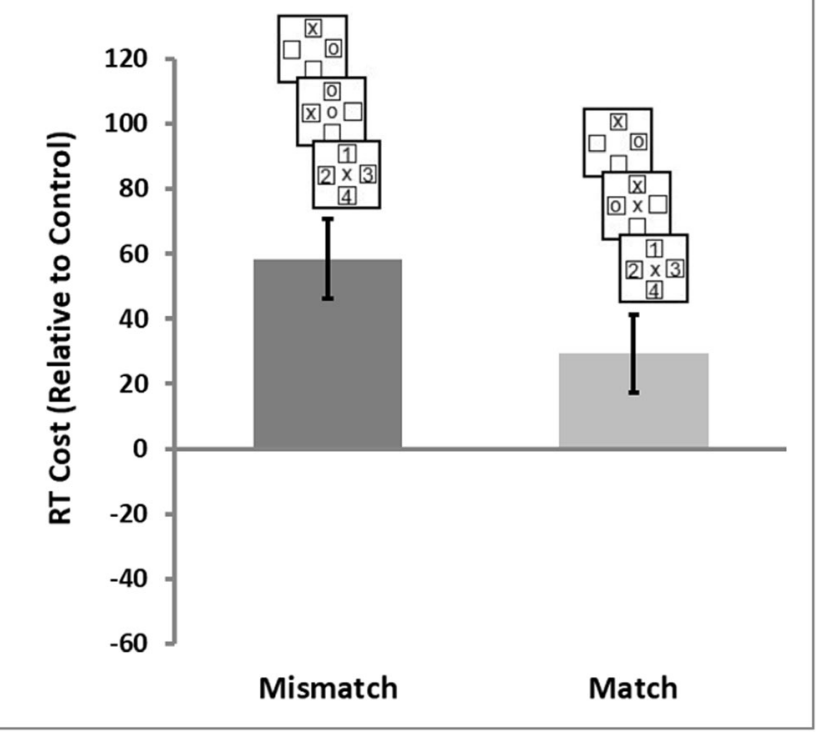

Fig. 3 Mean reaction time (RT) difference scores for Experiment 1. Error bars represent the standard errors of the mean difference scores. Match and mismatch refer to whether the spatially overlapping intervening display item matches or mismatches the item from the memory array

trials (Cali et al., 2015; Fiacconi \& Milliken, 2012, 2013). Although marginal, the RT cost associated with match trials in Experiment 1 was also of interest when considered in relation to well-established object-file updating principles. According to the object-file review principle, responses to matching targets in the intervening display ought to have been faster rather than slower when compared to the control condition (see Park \& Kanwisher, 1994). In Experiment 2, we turned our focus to understanding why these mnemonic and RT costs occurred for match trials.

The answer to this question may stem from some important methodological differences between our prior work and Experiment 1. In our prior studies, participants often knew in advance of each trial the identity of the intervening display target - in some cases they were to localize the target $\mathrm{O}$ in the intervening display on all trials (Fiacconi \& Milliken, 2012).

Table 4 Raw mean localization error rates for each condition in Experiments 1 and 2

\begin{tabular}{llll}
\hline Experiment & Control Trials & \multicolumn{2}{l}{ Experimental Trials } \\
\cline { 3 - 4 } & & Match & Mismatch \\
\hline 1 & 3.6 & 3.6 & 4.1 \\
2 & 6.5 & 4.5 & 7.4 \\
\hline
\end{tabular}

Note that the cue repeat/switch variable is not relevant here, given that localization error rates are measured for the intervening display only. Therefore, the relationship between the identity of the intervening display target and the subsequent memory probe is irrelevant for this measure. 
In contrast, in Experiment 1 of the present study, the identity of the intervening display target varied across trials and was disclosed only upon onset of the intervening display itself. Importantly, knowing the identity of the intervening display target at the outset of each trial could bias attention toward this item in both the memory array and the intervening display (Downing, 2000; Soto, Heinke, Humphreys, \& Blanco, 2005; Soto, Hodsoll, Rotshtein, \& Humphreys, 2008; Soto $\&$ Humphreys, 2007), and would alleviate the need for rapid target identification and consequent response selection processes otherwise required at onset of the intervening display. One possibility is that engagement of these selection processes when the intervening display appears may carve the memory array and the subsequent intervening display into discrete episodes (Wyble, Potter, Bowman, \& Nieuwenstein, 2011), obviating fluent integration even between location-identity repetitions on match trials. It follows that in the absence of such selection processes, object-file updating could be unmasked, in turn yielding accurate memory performance and fast responding for these trials. To test this hypothesis, in Experiment 2 we presented the identity of the intervening display target at the beginning of each trial prior to onset of the memory array.

\section{Method}

Participants The participants were 20 McMaster University undergraduate students (15 females, five males) who participated in exchange for course credit or financial compensation. The mean age of the participants was 20.7 years. All participants reported normal or corrected-to-normal vision.

Stimuli and apparatus These were the same as in Experiment 1.

Design The design of Experiment 2 was identical to that of Experiment 1.

Procedure The experimental procedure was similar to that of Experiment 1, with two exceptions. First, at the outset of each trial, the display of the four empty boxes appeared for $700 \mathrm{~ms}$, followed by presentation of the intervening display target cue for a duration of $300 \mathrm{~ms}$. Second, the ISI between the memory array and the intervening display was $500 \mathrm{~ms}$. The remainder of the trial unfolded in the same manner as in Experiment 1.

\section{Results and discussion}

The treatment of the data for Experiment 2 was identical to that in Experiment 1. In addition, we excluded from analysis the data from one participant whose mean overall memory accuracy (proportion correct) across all conditions was less than .30, leaving 19 participants eligible for the analysis.
Memory analyses As in Experiment 1, we included in our analysis only those trials on which a correct response was made to the intervening display target. The raw mean proportions correct for all four conditions are displayed in Table 2. The primary memory analyses were again conducted on the mnemonic costs for the two experimental conditions. The mean mnemonic costs, collapsed across participants, are displayed in Fig. 4.

Whereas we found a highly significant mnemonic cost for match trials in Experiment 1, there was no hint of such an effect in Experiment 2, $t(18)=0.30, p=.76, d_{\text {cohen }}=0.07$. This result is consistent with our hypothesis that presenting the intervening display target cue prior to the memory array increased the likelihood of object-file updating, which in turn benefited memory for match trials. In contrast to the stark change in memory performance for match trials across experiments, the results for the mismatch trials are near identical across Experiments 1 and 2 (cf. Figs. 2 and 4). As in Experiment 1, a robust mnemonic cost emerged on mismatch trials, $t(18)=5.00, p<.001, d_{\text {cohen }}=1.15$. The mnemonic cost for mismatch trials was also larger than that for the match trials, $t(18)=4.74, p<.001, d_{\text {cohen }}=1.74$.

To further solidify the disparity in the pattern of mnemonic costs across Experiments 1 and 2, we also analyzed the cost scores across experiments using a mixed analysis of variance (ANOVA) that treated experiment $(1,2)$ and condition (match,

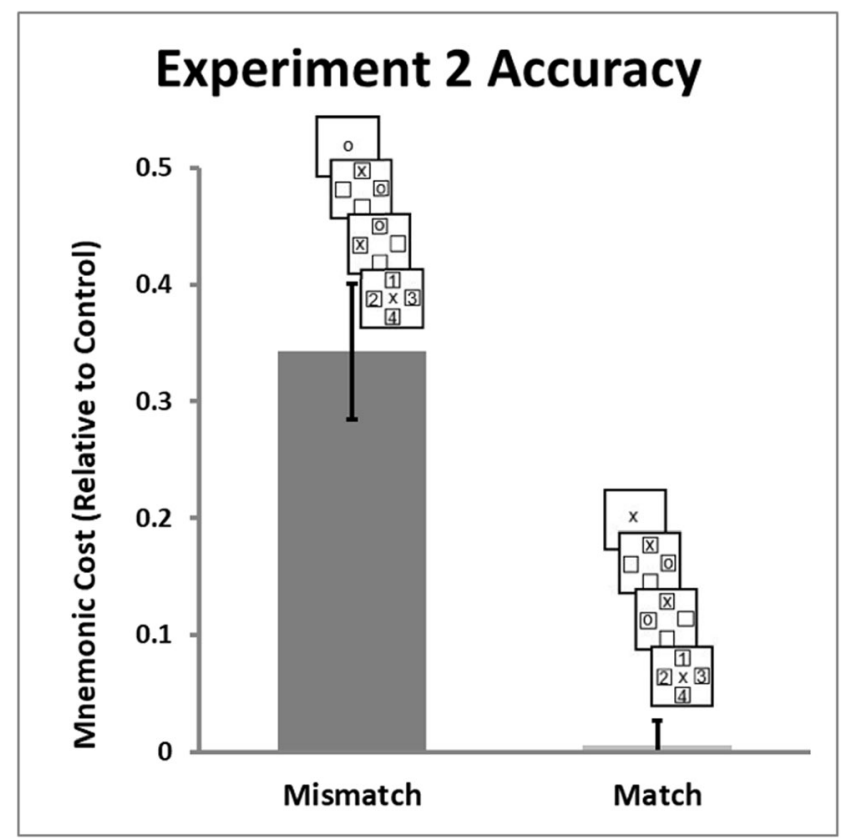

Fig. 4 Mean memory accuracy difference scores for each condition in Experiment 2. Error bars represent the standard errors of the mean difference scores. Trial diagrams are shown above each bar, where each large square represents a possible configuration for the target cue, memory array, intervening display, and test display in that trial type. Match and mismatch refer to whether the spatially overlapping intervening display item matches or mismatches the item from the memory array 
mismatch) as between- and within-subjects factors, respectively. As expected, the ANOVA yielded a significant interaction, $F(1,37)=7.68, p=.009, \eta_{\mathrm{p}}{ }^{2}=.17$. Subsequent simple main effect analyses revealed that the mnemonic cost for match trials in Experiment 1 was larger than that in Experiment $2, t(37)=4.29, p<.001, d_{\text {cohen }}=1.38$. In contrast, the mnemonic costs for mismatch trials did not differ across experiments, $t(37)=0.64, p=.52, d_{\text {cohen }}=0.21$.

To recap, the memory analyses across Experiments 1 and 2 demonstrate that cueing the identity of the intervening display target prior to the memory array affected mismatch trials minimally, whereas it eliminated the robust mnemonic cost for match trials that was observed in Experiment 1 (cf. Figs. 2 and 4). This result supports the idea that canonical object-file updating processes that produce fluent updating, and therefore accurate memory performance, for location-identity conjunction matches occurred in Experiment 2 but not in Experiment 1.

RT analyses Only trials on which participants responded correctly to the intervening display target were included in the RT analyses. The correct RTs for each condition were submitted to the same outlier procedure used in Experiment 1, which resulted in the exclusion of $3.0 \%$ of the RTs from further analysis. Mean RTs were computed from the remaining observations, and these raw mean RTs for each of the three conditions are displayed in Table 3 . Analyses were again conducted on the RT costs, as defined in Experiment 1. The mean RT costs for each condition, collapsed across participants, are depicted in Fig. $5 .^{3}$

A comparison of Figs. 3 and 5 reveals that the RT cost for match trials changed qualitatively across experiments. Whereas there had been an RT cost that approached significance in Experiment 1, there was an RT benefit that approached significance in Experiment 2, $t(18)=2.07, p=$ $.053, d_{\text {cohen }}=0.48$. In contrast to the results for the match condition, the results for the mismatch condition were broadly similar across experiments; as in Experiment 1, we found a robust RT cost in Experiment 1, $t(18)=4.75, p<.001, d_{\text {cohen }}=$ 1.09. Accordingly, the RT cost for mismatch trials was significantly greater than the RT cost score for match trials, $t(18)=$ $4.60, p<.001, d_{\text {cohen }}=1.50$.

As was done for the mnemonic costs, we also compared directly the magnitudes of the RT costs for match and mismatch trials across experiments, using a mixed ANOVA that included experiment $(1,2)$ and condition (match, mismatch) as between- and within-subjects factors, respectively. Once again, the ANOVA yielded a significant interaction between

\footnotetext{
$\overline{3}$ The error rates for intervening-display responses (see Table 3 ) were analyzed similarly to the RT data. We observed a significant reduction in errors for the match relative to the control conditions, $t(18)=2.12, p=.048, d_{\text {cohen }}=0.49$, which aligns with the RT results.
}

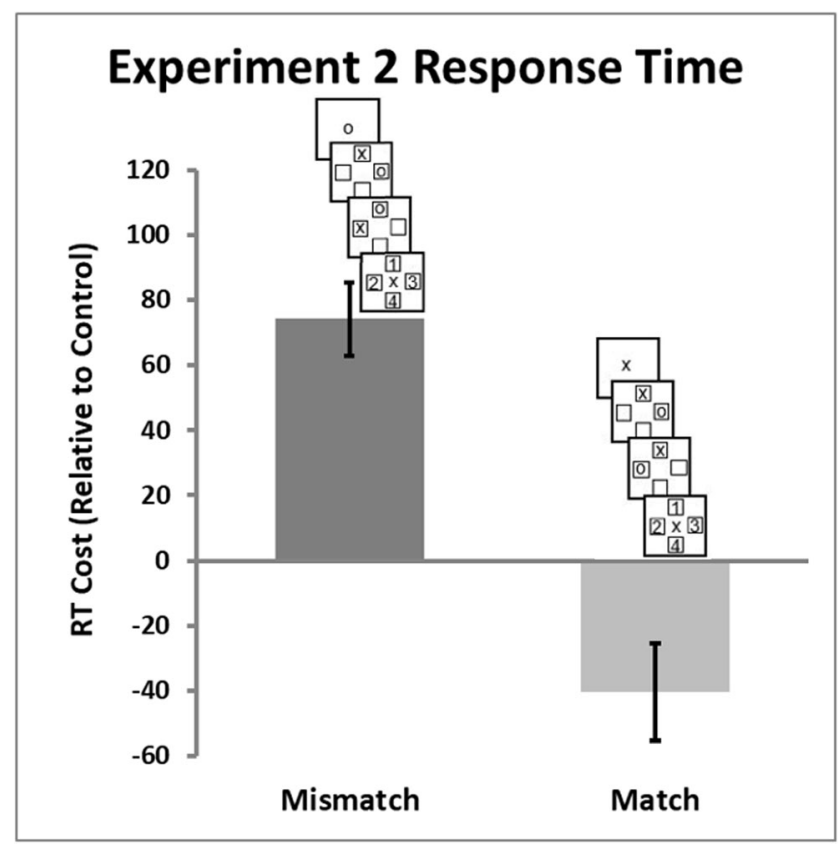

Fig. 5 Mean reaction time (RT) difference scores for each condition in Experiment 2. Error bars represent the standard errors of the mean difference scores. Match and mismatch refer to whether the spatially overlapping intervening display item matches or mismatches the item from the memory array

these factors, $F(1,37)=6.84, p=.01, \eta_{\mathrm{p}}{ }^{2}=.16$. This interaction was driven largely by the difference in RT costs for match trials across experiments, as the RT cost for match trials in Experiment 1 was larger than that in Experiment 2, $t(37)=$ $2.77, p=.009, d_{\text {cohen }}=0.89$. In contrast, the RT costs for mismatch trials did not differ across experiments, $t(37)=$ $0.71, p=.48, d_{\text {cohen }}=0.23$.

Summary The key result in the memory analyses was that the mnemonic cost for match trials observed in Experiment 1 was eliminated in Experiment 2. The key result in the RT analyses was that the RT cost for match trials observed in Experiment 1 turned into an RT benefit in Experiment 2. These results are consistent with the proposal that presentation of the intervening display target cue prior to the memory array promoted canonical object-file updating processes. By this view, increased object-file updating on match trials in Experiment 2 relative to Experiment 1 both speeded responding and improved memory performance. This striking correspondence between RT performance and memory accuracy has interesting implications for the object/event-file updating framework.

\section{General discussion}

Our goal in this study was to examine the extent to which object-file updating processes guide not only speeded performance, but can also simultaneously shape VWM accuracy- 
two fields in which Anne Treisman left an immutable legacy. To address this issue, the present study employed an experimental procedure that allowed for the concurrent examination of both measures in a single task.

In Experiment 1, we found parallel mnemonic and RT costs when participants responded to a target item in the intervening display that appeared in the same spatial location as the subsequently probed item from the memory array. That is, memory for the location of an item that was spatially overlapped in the intervening display was impaired, and RT to the overlapping target item was slowed. Particularly intriguing is that this effect occurred even when the target in the intervening display was identical to the memory array item tested. This finding supports the idea that mnemonic and RT costs are not limited to mismatch trials, and can in fact occur when the probed item is subsequently overlapped by the same identity if a response is directed to this subsequent item. This finding also weighs against the notion that location-identity conjunction matches between successive displays always trigger object-file updating processes that lead to fast target responses.

In Experiment 2, we examined whether the mnemonic and RT costs for match trials observed in Experiment 1 hinge on the time at which the identity of the intervening display target was disclosed. In fact, when this target item was cued at the beginning of each trial, the mnemonic cost on match trials observed in Experiment 1 was eliminated in Experiment 2. Moreover, the pattern of RTs to intervening targets mirrored that of the memory accuracy data. Whereas an RT cost was observed for match trials in Experiment 1, this effect reversed to an RT benefit in Experiment 2. Together, these results highlight a strong correspondence between patterns of VWM performance and RT performance, and point to the possibility of a common mechanism underlying these two behavioural effects.

We have suggested in our prior work (Cali et al., 2015; Fiacconi \& Milliken, 2012, 2013) that the object-file framework of Kahneman and colleagues (Kahneman et al., 1992) may be useful in explaining mnemonic costs for mismatch trials. By this view, responding to a new identity at a previously occupied location may forcefully dissolve the previously stored location-identity binding in VWM, and lead to the formation of a new location-identity binding that overwrites the previous binding and leaves it unavailable for report. Although this account can accommodate many of the results reported here and elsewhere (Fiacconi \& Milliken, 2012, 2013), it does not readily accommodate the mnemonic cost observed for match trials in Experiment 1. According to the object-file updating framework, location-identity matches should lead to fluent updating such that current perceptual input is integrated with an existing object file. Such integration would be expected to facilitate RT for match trials (Hommel, 1998; Kahneman et al., 1992; Milliken, Tipper, \& Weaver, 1994; Park \& Kanwisher, 1994) and perhaps to enhance VWM performance. Clearly, this prediction was not borne out in Experiment 1. Instead, we observed costs for both RT and memory accuracy. These findings contrast with those of Experiment 2, in which the observed benefits to performance and memory for match trials falls perfectly in line with the predictions of the object-file updating framework. We now turn to a discussion of the mechanisms that might be responsible for the discrepancy in results across Experiments 1 and 2.

\section{Endogenous control of event integration}

To account for these results, we propose that object-file updating in the traditional sense may not be an obligatory process, and that it can be subject to endogenous control that depends on the demands of the task. The idea that we can exert control over the integration of perceptual events has gained increasing support in the literature (Akyürek, Schubö, \& Hommel, 2012; Akyürek, Toffanin, \& Hommel, 2008; Bowman \& Wyble, 2007; Wyble et al., 2011), and this concept has been used to help explain a range of different experimental phenomena including the attentional blink (Akyürek et al., 2012; Akyürek et al., 2008; Bowman \& Wyble, 2007; Wyble et al., 2011), whole report (Nieuwenstein \& Potter, 2006), and repetition blindness (Kanwisher, 1987; Mozer, 1989; Wyble et al., 2011). Applied to the present set of experiments, it is possible that by withholding the identity of the target item until onset of the intervening display in Experiment 1 , participants were forced to endogenously segregate the memory array and intervening display events to encode and successfully localize the target item in the latter display. Put differently, the requirement to attend selectively to the intervening display to identify the target item and subsequently respond to its location may have temporally segregated the trial into two discrete attentional "episodes" (Wyble et al., 2011). According to Wyble et al., the purpose of dividing perceptual input into discrete temporal chunks is to encode each chunk into working memory as a unique episodic representation - that is, to encode each event within a unique spatiotemporal context that distinguishes it from neighboring input. Therefore, such an encoding process involves binding some visual content (e.g., O), referred to as a "type," with some spatial or temporal index (e.g., top) to create a unique instance, referred to as a "token" (e.g., O, top). In the case of a match trial on which participants respond to the same item in the same location, endogenously segregating the memory array and the intervening display may force a "re-tokenization" process in which the type contained within the memory array and stored in memory (e.g., $\mathrm{O}$ ) has to be re-encoded in the intervening display as a distinct event bound to a unique spatiotemporal context. If one assumes that the same spatial index (e.g., top) can only be used to "tokenize" one type at a time, then re-tokenizing the target in the intervening display (e.g., O) with the same spatial index (e.g., top) may displace the 
previous instance involving this index from working memory, leading to poor memory performance.

In contrast, by providing the identity of the target for the intervening display at the outset of each trial prior to the memory array in Experiment 2, participants were able to hold in mind a particular identity during encoding of the memory array, which likely biased their attention toward this item during encoding (Downing, 2000; Soto et al., 2005; Soto et al., 2008; Soto \& Humphreys, 2007). Such biasing might make a subsequent location-identity repetition in the intervening display particularly salient, allowing participants to compensate for the otherwise deleterious mnemonic consequences of responding to a location-identity match. Another way to conceptualize the influence of this manipulation is to assume that holding the target item in working memory may have promoted event integration (i.e., fluent object-file updating) between the memory array and the intervening display, as participants no longer must disengage their focus from maintaining items in memory at onset of the intervening display to identify the target identity. Eliminating this selection requirement at onset of the intervening display might allow the perceptual system to bridge these two events, such that they are encoded together in the same attentional episode (Wyble et al., 2011), leaving memory for the original binding intact. Although the present set of data does not allow us to adjudicate between these alternative interpretations, they do point to an interesting avenue for further research. Regardless of the interpretation of this result, our findings provide a novel and compelling demonstration that under some experimental conditions, visual short-term memory for the location of a given item can be poor when that same item is repeated in the same location a short time later.

Interestingly, the concept of perceptual event integration/ segregation has also been invoked to account for RT data in the performance domain (Lupiáñez, 2010; Lupiáñez, Milliken, Solano, Weaver, \& Tipper, 2001). Much like in the present experiments, the influence of location-identity matches on RT has produced somewhat equivocal results, with some studies reporting response facilitation (Kahneman et al., 1992; Park \& Kanwisher, 1994), and yet others reporting response slowing (Hu, Samuel, \& Chan, 2011; Lupiáñez, 2010; Milliken et al., 1994; Tipper, Weaver, \& Milliken, 1995). To resolve these contradictory findings, it has been proposed that task demands can dictate the extent to which the perceptual system retrieves the previous location-identity binding to aid performance (Lupiáñez, 2010; Milliken, Tipper, Houghton, \& Lupiáñez, 2000). When the task demands require the distinct encoding of two separate events, repetition can produce interference by serving as a bottom-up cue for integration that conflicts with the topdown mental set of attempting to encode each event as a unique instance. This conflict, in turn, produces slower response to location-identity matches, and has sometimes been referred to as a "detection cost" (Lupiáñez, 2010). By contrast, when the task demands allow for the retrieval of the previous location-identity binding, such a repetition results in a fluent updating process in which repeated perceptual information is readily integrated with a preexisting object file, and performance is facilitated. This account is very similar in spirit to our explanation for the observed mnemonic costs associated with match trials. Indeed, one could conceive of "retokenizing" as one mechanism by which we encode each event as a unique episode. Our data suggest that the process of "re-tokenizing" may not only have mnemonic consequences, but also may be associated with costs in perceiving and responding to perceptual input when information is repeated, serving as a common mechanism driving both memory performance and RT in the present experiments. We note that although processes related to event integration have received extensive consideration in the attention and performance literature (Akyürek et al., 2013; Akyürek et al., 2008; Bowman, \& Wyble, 2007; Hommel, 1998; Kahneman et al., 1992; Lupiáñez, 2010; Lupiáñez et al., 2001; Milliken et al., 1994; Park \& Kanwisher, 1994; Wyble et al., 2011), they have rarely been examined in the context of visual short-term memory. The present findings suggest a novel synergy between these literatures, highlighting a common principle that is potentially operative in both domains.

\section{Relation to the previous literature}

Visual short-term memory (VWM) In linking event integration processes commonly invoked in the performance literature with mnemonic consequences in the visual memory domain, our results shed new light on the factors that govern the remembering of visual information over the short-term. In particular, we would like to emphasize that standard change detections tasks commonly used to study the properties of VWM representations may not capture how VWM is utilized in dynamic environments that present a continuous stream of visual input and myriad task goals. Indeed, under these circumstances the principles that govern VWM may diverge from those revealed by performance variations in change detection tasks. Indeed, the present findings demonstrate that even when memory for the feature conjunctions of only two items must be maintained, responding to new visual input in which some features are re-paired yields a dramatic impairment in memory for the original feature conjunction (see also Cali et al., 2015; Fiacconi \& Milliken, 2012, 2013). Such a result is not readily captured by models of VWM that are designed to account for performance in relatively "static" change detection tasks, and point to the potential importance of event integration processes that co-ordinate the interaction between stored information and new visual input. Accordingly, the patterns of memory performance observed here suggest that our understanding of the principles that guide our ability to remember visual 
information over the short-term may profit from an increased focus on how we co-ordinate ongoing perception and action with information stored in VWM.

Binding actions and perceptual features A key feature of our experiments was that participants were required to respond to the location of a target item in the second (i.e., intervening display) of two successive displays, while simultaneously holding in memory the locations of items from the preceding memory array. Notably, this procedural sequence bears a considerable resemblance to other well-known procedures designed to probe the integration between perceptual features and response codes (Hommel, 1998; Stoet \& Hommel, 1999). Of particular relevance here is the ABBA procedure introduced by Stoet and Hommel, in which participants were shown a stimulus (e.g., A) and told to plan a specific response (e.g., left) based on the identity of this stimulus but not to execute it until it reappeared at a later time. After planning this response, a second stimulus (e.g., B) appeared and participants made a response (e.g., left) again based on its identity. Following the response to the B stimulus, the A stimulus then reappeared, and participants executed the previously planned response. The key aspect of this design is that the responses made to each stimulus could be manipulated orthogonally such that response code generated for B may or may not share a feature in common with the previously planned response code for A, affording the opportunity to examine how response feature overlap affects performance. Critically, it was found that when the response code planned for stimulus A shared features with the subsequently generated response code for stimulus B, response time to execute the response to B was slowed. This result was interpreted as demonstrating that a given response feature can only be bound to one stimulus at a time, and that a response to another stimulus involving that same feature entails a time-consuming rebinding process.

In many respects, the procedures used in the present study are quite similar to those used by Stoet and Hommel (1999). One could reasonably conceive of memorizing the location of each item in the memory array as "planning" a response that is to be executed at the time of the test display. Moreover, responding to the location of the intervening display target could be considered analogous to responding to the B stimulus in the Stoet and Hommel procedure. According to the account forwarded by Stoet and Hommel, insofar as the target in the intervening display target shares the same response with the subsequently probed item from the memory array (e.g., "top"), responses to the intervening display target ought to be slowed. Indeed, this was exactly the pattern of RT results we obtained in Experiment 1. By this view, the re-binding processes needed to link the same response feature with a different stimulus appears not only to produce performance costs (e.g., slowed responses to the second stimulus), but mnemonic costs as well (i.e., poor memory for the response- relevant feature of the memory array item; i.e., location). Extending this analogy to Experiment 2, it is possible that providing the identity for the intervening display prior to the memory array was somewhat akin to telling participants what response to make to stimulus B prior to its appearance. Under these conditions, the rebinding processes needed to conjoin the same response code to different stimuli may not be necessary. As such, the costs associated with re-binding would be eliminated. We note that these re-binding processes thought to underlie the performance costs and potentially also the mnemonic costs observed here could be similar to the "retokenization" process that was outlined above. Subsequent work will be needed to further explore how response binding processes interact with the contents of VWM.

\section{Conclusion}

An important contribution of the work presented here is to suggest the possibility that processes hypothesized to play a role in guiding speeded performance may also have potent consequences for VWM accuracy. This work speaks to the importance of VWM dynamics and emphasizes the influence of control processes that function to organize the interaction between current visual input and short-term memory representations. As such, the findings reported here point to an intriguing link between processes known to influence behavior in attention and performance tasks, and those that shape VWM performance in dynamic contexts.

Open Practices Statement None of the data or materials for the experiments reported here are available, and none of the experiments was preregistered. However, the data and analysis materials are available from the first author upon request.

\section{References}

Akyürek, E. G., Schubö, A., \& Hommel, B. (2013). Attentional control and competition between episodic representations. Psychological Research, 77, 492-507. doi:https://doi.org/10.1007/s00426-0120445-9

Akyürek, E. G., Toffanin, P., \& Hommel, B. (2008). Adaptive control of event integration. Journal of Experimental Psychology: Human Perception and Performance, 34, 569-577.

Allen, R. J., Baddeley, A. D., \& Hitch, G. J. (2006). Is the binding of visual features in working memory resource-demanding? Journal of Experimental Psychology: General, 135, 298-313. doi:https://doi. org/10.1037/0096-3445.135.2.298

Alvarez, G. A., \& Thompson, T. W. (2009). Overwriting and rebinding: Why feature-switch detection tasks underestimate the binding capacity of visual working memory. Visual Cognition, 17, 141-159. doi:https://doi.org/10.1080/13506280802265496

Averbach, E., \& Coriell, A. S. (1961). Short-term memory in vision. Bell Systems Technical Journal, 40, 309-328. 
Bovens, N., \& Brysbaert, M. (1990). IBM PC/XT/AT and PS/2 Turbo Pascal timing with extended resolution. Behavior Research Methods, Instruments, \& Computers, 22, 332-334. doi:https://doi. org/10.3758/BF03209826

Bowman, H., \& Wyble, B. (2007). The simultaneous type, serial token model of temporal attention and working memory. Psychological Review, 114, 38-70. doi:https://doi.org/10.1037/0033-295X.114.1. 38

Cali, J. N., Fiacconi, C. M., \& Milliken, B. (2015). A response binding effect in visual short-term memory. Visual Cognition, 23, 489-515.

Delvenne, J.-F., \& Bruyer, R. (2004). Does visual short-term memory store bound features? Visual Cognition, 11, 1-27. doi:https://doi. org/10.1080/13506280344000167

Downing, P. E. (2000). Interactions between visual working memory and selective attention. Psychological Science, 11, 467-473. doi:https:// doi.org/10.1111/1467-9280.00290

Fiacconi, C. M., \& Milliken, B. (2012). Contingency blindness: Location-identity binding mismatches obscure awareness of contingencies and produce profound interference in visual working memory. Memory \& Cognition. 40, 932-945.

Fiacconi, C. M., \& Milliken, B. (2013). Visual memory for feature bindings: The disruptive effect of responding to new perceptual input. Quarterly Journal of Experimental Psychology, 66, 1572-1600. doi: https://doi.org/10.1080/17470218.2012.753925

Gajewski, D. A., \& Brockmole, J. R. (2006). Feature bindings endure without attention: Evidence from an explicit recall task. Psychonomic Bulletin \& Review, 13, 581-587. doi:https://doi.org/ 10.3758/BF03193966

Gorgoraptis, N., Catalao, R. F. G., Bays, P. M. and Husain, M. (2011). Dynamic updating of working memory resources for visual objects. Journal of Neuroscience, 31, 8502-8511. doi:https://doi.org/10. 1523/JNEUROSCI.0208-11.2011

Hollingworth, A., \& Rasmussen, I. P. (2010). Binding objects to locations: The relationship between object files and visual working memory. Journal of Experimental Psychology: Human Perception and Performance, 36, 543-564. doi:https://doi.org/10.1037/ a0017836

Hommel, B. (1998). Event files: Evidence for automatic integration of stimulus-response episodes. Visual Cognition, 5, 183-216. doi: https://doi.org/10.1080/713756773

Hu, F. K., Samuel, A. G., \& Chan, A. S. (2011). Eliminating inhibition of return by changing salient nonspatial attributes in a complex environment. Journal of Experimental Psychology: General, 140, 3550. doi:https://doi.org/10.1037/a0021091

Johnson, J. S., Hollingworth, A., \& Luck, S. J. (2008). The role of attention in the maintenance of feature bindings in visual short-term memory. Journal of Experimental Psychology: Human Perception and Performance, 34, 41-55. doi:https://doi.org/10.1037/00961523.34.1.41

Kahneman, D., Treisman, A., \& Gibbs, B. J. (1992). The reviewing of object files: Object-specific integration of information. Cognitive Psychology, 24, 175-219. doi:https://doi.org/10.1016/00100285(92)90007-O

Kanwisher, N. G. (1987). Repetition blindness: Type recognition without token individuation. Cognition, 27, 117-143. doi:https://doi.org/10. 1016/0010-0277(87)90016-3

Luck, S. J., \& Vogel, E. K. (1997). The capacity of visual working memory for features and conjunctions. Nature, 390, 279-281. doi:https:// doi.org/10.1038/36846

Lupiáñez, J. (2010). Inhibition of return. In A. C. Nobre \& J. T. Coull (Eds.), Attention and time (pp. 17-34). New York, NY: Oxford University Press.

Lupiáñez, J., Milliken, B., Solano, C., Weaver, B., \& Tipper, S. P. (2001). On the strategic modulation of the time course of facilitation and inhibition of return. Quarterly Journal of Experimental Psychology, 54A, 753-773. doi:https://doi.org/10.1080/02724980042000453
Makovski, T., Sussman, R., \& Jiang, Y. V. (2008). Orienting attention in visual working memory reduces interference from memory probes. Journal of Experimental Psychology: Learning, Memory, and Cognition, 34, 369-380. doi:https://doi.org/10.1037/0278-7393.34. 2.369

Makovski, T., Watson, L. M., Koutstaal, W., \& Jiang, Y. V. (2010). Method matters: Systematic effects of testing procedure on visual working memory sensitivity. Journal of Experimental Psychology: Learning, Memory, and Cognition, 36, 1466-1479. doi:https://oi. org/10.1037/a0020851

Milliken, B., Tipper, S. P., \& Weaver, B. (1994). Negative priming in a spatial localization task: Feature mismatching and distractor inhibition. Journal of Experimental Psychology: Human Perception \& Performance, 20, 624-646.

Milliken, B., Tipper, S. P., Houghton, G., \& Lupiáñez, J. (2000). Attending, ignoring, and repetition: On the relation between negative priming and inhibition of return. Perception \& Psychophysics, 62, 1280-1296. doi:https://doi.org/10.3758/BF03212130

Mozer, M. C. (1989). Types and tokens in visual letter perception. Journal of Experimental Psychology: Human Perception and Performance, 15, 287-303.

Nieuwenstein, M. R., \& Potter, M. C. (2006). Temporal limits of selection and memory encoding: A comparison of whole versus partial report in rapid serial visual presentation. Psychological Science, 17, 471475. doi:https://doi.org/10.1111/j.1467-9280.2006.01730.x

Park, J., \& Kanwisher, N. (1994). Negative priming for spatial locations: Identity mismatching, not distractor inhibition. Journal of Experimental Psychology: Human Perception and Performance, 20, 613-623. doi:https://doi.org/10.1037/0096-1523.20.3.613

Pertzov, Y., Dong, M. Y., Peich, M.-C., \& Husain, M. (2012). Forgetting what was where: The fragility of object-location binding. PLoS ONE, 7, e48214. doi:https://doi.org/10.1371/journal.pone.0048214

Saiki, J. (2003). Spatiotemporal characteristics of dynamic feature binding in visual working memory. Vision Research, 43, 2107-2123.

Soto, D., Heinke, D., Humphreys, G. W., \& Blanco, M. J. (2005). Early, involuntary top-down guidance of attention from working memory. Journal of Experimental Psychology: Human Perception and Performance, 31, 248-261. doi:https://doi.org/10.1037/0096-1523. 31.2.248

Soto, D., Hodsoll, J., Rotshtein, P., \& Humphreys, G. W. (2008). Automatic guidance of attention from working memory. Trends in Cognitive Sciences, 12, 342-348. doi:https://doi.org/10.1016/j.tics. 2008.05.007

Stoet, G., \& Hommel, B. (1999). Action planning and the temporal binding of response codes. Journal of Experimental Psychology: Human Perception and Performance, 25, 1625-1640.

Soto, D., \& Humphreys, G. W. (2007). Automatic guidance of visual attention from verbal working memory. Journal of Experimental Psychology: Human Perception and Performance, 33, 730-737. doi:https://doi.org/10.1037/0096-1523.33.3.730

Tipper, S. P., Weaver, B., \& Milliken, B. (1995). Spatial negative priming without mismatching: Comment on Park and Kanwisher (1994). Journal of Experimental Psychology: Human Perception and Performance, 21, 1220-1229. doi:https://doi.org/10.1037/00961523.21.5.1220

Treisman, A. M., \& Gelade, G. (1980). A feature-integration theory of attention. Cognitive Psychology, 12, 97-136. doi:https://doi.org/10. 1016/0010-0285(80)90005-5

Treisman, A., \& Zhang, W. (2006). Location and binding in visual working memory. Memory \& Cognition, 34, 1704-1719. doi:https://doi. org/10.3758/BF03195932

Ueno, T., Allen, R. J., Baddeley, A. D., Hitch, G. J., \& Saito, S. (2011a). Disruption of visual feature binding in working memory. Memory \& Cognition, 39, 12-23. doi:https://doi.org/10.3758/s13421-0100013-8 
Ueno, T., Mate, J., Allen, R. J., Hitch, G. J., \& Baddeley, A. D. (2011b). What goes through the gate? Exploring interference with visual feature binding. Neuropsychologia, 49, 1597-1604.

Van Selst, M., \& Jolicœur, P. (1994). A solution to the effect of sample size on outlier elimination. Quarterly Journal of Experimental Psychology, 47A, 631-650. doi:https://doi.org/10.1080/ 14640749408401131

Vogel, E. K., Woodman, G. F., \& Luck, S. J. (2001). Storage of features, conjunctions, and objects in visual working memory. Journal of Experimental Psychology: Human Perception and Performance, 27, 92-114. doi:https://doi.org/10.1037/0096-1523.27.1.92

Wheeler, M. E., \& Treisman, A. M. (2002). Binding in short-term visual memory. Journal of Experimental Psychology: General, 131, 4864. doi:https://doi.org/10.1037/0096-3445.131.1.48
Wyble, B., Potter, M. C., Bowman, H., \& Nieuwenstein, M. (2011). Attentional episodes in visual perception. Journal of Experimental Psychology: General, 140, 488-505. doi:https://doi.org/10.1037/ a0023612

$\mathrm{Xu}$, Y. (2002). Limitations of object-based feature encoding in visual short-term memory. Journal of Experimental Psychology: Human Perception and Performance, 28, 458-468.

Publisher's note Springer Nature remains neutral with regard to jurisdictional claims in published maps and institutional affiliations. 